\title{
Sarcomatoid Carcinoma: A Rare and unusual histological variant of prostatic carcinoma
}

\author{
Amit Gupta1, Bhavesh Chaddha', Puneet Prakash', Priya Pathak', Neelam Wadhwa² \\ ${ }^{1}$ Dept of Surgery, ${ }^{2}$ Dept of Pathology, University College of Medical Sciences and Guru Teg Bahadur Hospital, New Delhi, India
}

\section{A B S TR A C T}

Carcinosarcoma of Prostate also known as Sarcomatoid carcinoma is a rare biphasic malignancy with a malignant epithelial component (carcinomatous) and a malignant mesenchymal component. We report a case of this rare and unusual histological variant of prostatic carcinoma.

Key Words: Sarcomatoid, Prostate specific antigen, Prostate carcinoma
Access this article online Website:

http://nepjol.info/index.php/AJMS

DOI: 10.3126/ajms.v6i5.12336

\section{INTRODUCTION}

Malignant tumors of the prostate that display biphasic patterns are very rare. They include sarcomatoid carcinomas (SC) and carcinosarcomas (CS). It is an admixture of high grade epithelial and sarcomatoid components, although it is still unclear that these cases represent a co-existent epithelial and mesenchymal elements (carcinosarcoma) or an evolution of an underlying adenocarcinoma into a lesion with associated sarcomatoid features and occasional heterologous elements (sarcomatoid carcinoma). ${ }^{1}$ However, WHO classification of urinary tract tumor does not distinguish between Sarcomatoid carcinoma (SC) and Carcinosarcoma (CS) and mentions all these lesions under the term "Sarcomatoid carcinoma". In contrast to adenocarcinoma Prostate, it is an aggressive malignancy, while the treatment is non-standardized. The patient may present with irritative symptoms (frequency, nocturia, urgency) and/or obstructive symptoms (poor stream, incomplete emptying or retention). Hematuria, perineal/ rectal pain and burning on ejaculation are uncommon symptoms. ${ }^{2}$ Hereby, we report a case of a 65 year old gentleman with Sarcomatoid carcinoma Prostate and discuss the treatment protocol of this uncommon tumor.

\section{Case report}

A 65 year old gentleman presented to our department with complaints of increased frequency of urine for last
6 months and back ache for the last two months. Digital rectal examination revealed grade II prostatomegaly, with left lobe of Prostate disproportionately enlarged and hard in consistency. There was no palpable lymphadenopathy, and the rest of his physical examination was unremarkable. His serum PSA was $495 \mathrm{ng} / \mathrm{ml}$. A tru-cut biopsy of Prostate showed malignant epithelial cells forming glands (Adenocarcinoma) scattered in a stroma composed of bizarre mesenchymal cells (Sarcoma) with infiltration into adipose tissue consistent with diagnosis of Carcinosarcoma Prostate (Gleason score 4+5) (Figures 1 and 2).

In view of biopsy findings patient underwent further evaluation and found to have bone metastases. Patient was started on anti-androgen therapy and bilateral subcapsular Orchiectomy was done. Palliative External beam Radiotherapy was recommended to painful bone metastases along with Inj Bisphosphonates monthly.

\section{DISCUSSION}

Carcinosarcoma of Prostate also known as Sarcomatoid carcinoma is a rare biphasic malignancy with a malignant epithelial component (carcinomatous) and a malignant mesenchymal component (sarcomatous) with the presence or absence of heterologous elements. ${ }^{3,4}$ The histogenesis of prostate carcinosarcoma is not completely understood. Proposed mechanisms include transformation of 


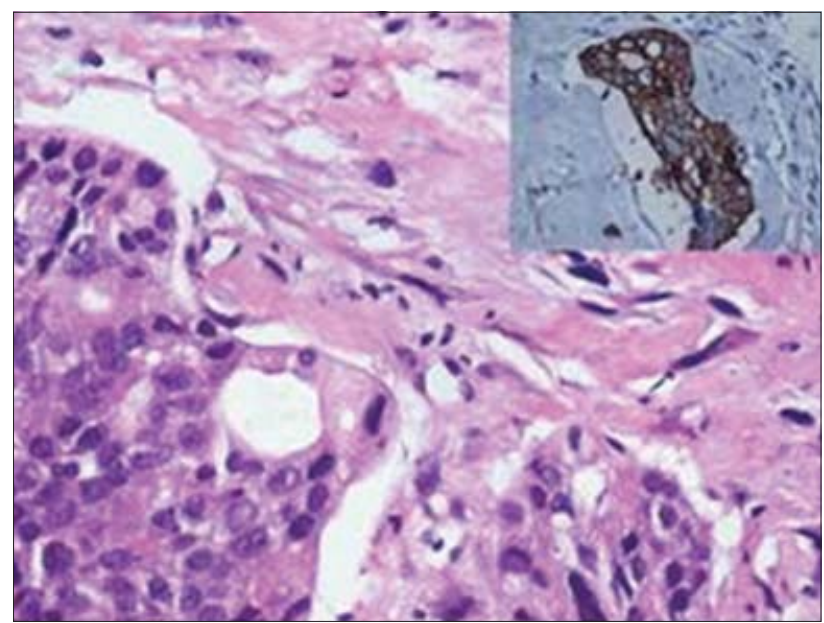

Figure 1: High grade adenocarcinoma admixed with malignant spindle cells (Hematoxylin Eosin stain X 400). Inset: Carcinomatous element is strongly positive for cytokeratin (cytokeratin immunohistochemistry $x$ 100).

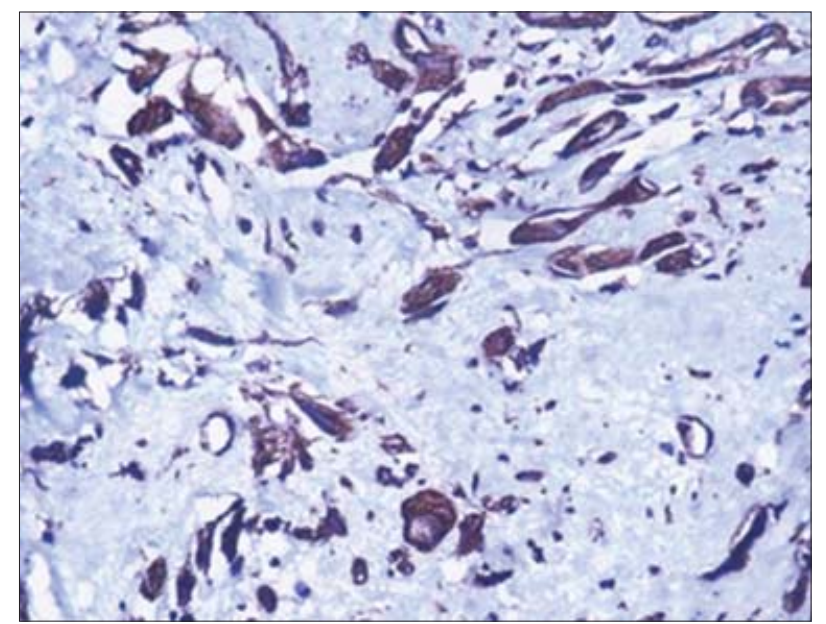

Figure 2: Malignant Spindle cells showing vimentin positivity (immunohistochemistry for vimentin $\mathrm{x} 400$ ).

epithelial elements into a sarcomatous component and divergent differentiation of epithelial stem cells into both malignant components. WHO classification use the term "Sarcomatoid carcinoma" to denote all of these lesions. ${ }^{5}$ The carcinomatous component is usually acinar but may be ductal adenocarcinoma. ${ }^{6}$ Squamous or adenosquamous carcinoma ${ }^{7}$ or mixed urothelial squamous components ${ }^{8}$ and is usually of high grade. ${ }^{9}$ The type of sarcoma may vary, including elements such as osteosarcoma, chondrosarcoma, rhabdomyosarcoma, leiomyosarcoma and angiosarcoma. In about half of all cases, the initial diagnosis was a usual acinar adenocarcinoma, followed by hormonal and/or radiation therapy, with a subsequent diagnosis of sarcomatoid carcinoma. By immunohistochemistry, epithelial elements react with cytokeratins, PSA, PSAP whereas sarcomatoid elements react with vimentin or specific markers corresponding to the mesenchymal differentiation. ${ }^{10}$ The sarcomatoid component is considered to be Gleason grade 5, with the glandular element, if present, assigned a grade according to the usual rules. PSA values are usually within normal limits, possibly due to "dedifferentiation" of tumor cells. ${ }^{8}$

The prognosis of prostate carcinosarcoma is poor, with death invariably occurring within 2 years regardless of treatment modality.

\section{REFERENCES}

1. Grignon DJ. Unusual subtypes of prostate cancer. Mod Pathol 2004;17(3):316-327.

2. Nazeer T, Barada JH, Fisher HAG and Ross JS. Prostatic carcinosarcoma: case report and review of literature. J Urol 1991; 146: 1370.

3. Mostofi FK and Price EB. Atlas of Tumor Pathology: Tumors of Male Genital System, series 2, part 8. Washington, DC: Armed Forces Institute of Pathology; 1973. Malignant tumors of the prostate; pp. 257-258.

4. Hansel DE and Epstein JI. Sarcomatoid carcinoma of the prostate: a study of 42 cases. Am J Surg Pathol 2006;30:13161321.

5. World Health Organization Classification of Tumors, editor. Lyon: Pathology and Genetics of Tumors of the Urinary System and Male Genital Organs. IARC Press; 2004

6. PobletE, Gomez-Tierno Aand Alfaro L. Prostatic carcinosarcoma: a case originating in a previous ductal adenocarcinoma of the prostate. Pathol Res Pract 2000; 196:569-572.

7. Berney DM, Ravi R and Baithun SI. Prostatic carcino-sarcoma with squamous cell differentiation: a consequence of hormonal therapy. Report of two cases and review of the literature. J Urol Pathol 1979;11:123-132.

8. Rogers CG, Parwani A, Tekes A, Schoenberg MP and Epstein JI. Carcinosarcoma of the prostate with urothelial and squamous components. J Urol 2005; 173:439-440.

9. Mazzucchelli R1, Lopez-Beltran A, Cheng L, Scarpelli M, Kirkali $Z$ and Montironi R. Rare and unusual histological variants of prostatic carcinoma: clinical significance. BJU Int 2008;102(10):1369-1374.

10. Wick MR, Brown BA, Young RH and Mills SE. Spindle-cell proliferations of the urinary tract: an immu-nohistochemical study. Am J Surg Pathol 1988;12:379.

\footnotetext{
Authors Contribution:

AG \& BC - operated the case, Pu P-collection of photographs; AG- compiled the case report; NW \& PP - pathological content of case report.

Source of Support: Nil, Conflict of Interest: None declared.
} 